\title{
Das Institutionelle Abkommen Schweiz-EU - ein Fall für das obligatorische Staatsvertragsreferendum?
}

\section{Andreas Glaser *}

Nach dem geplanten EWR-Beitritt im Jahr 1992 und dem Beitritt zu Schengen/Dublin im Jahr 2007 stellt sich mit Blick auf das Institutionelle Abkommen Schweiz-EU (InstA) erneut die Frage, ob der betreffende Genehmigungsbeschluss den Beitritt zu einer supranationalen Gemeinschaft gemäss Art. 140 Abs. 1 Bst. b BV bewirkt und dieser somit dem obligatorischen Referendum zu unterstellen ist. Der Beitrittsbeschluss zum EWR wurde seinerzeit aufgrund des Verfassungsrangs verschiedener Bestimmungen im Abkommen dem obligatorischen Staatsvertragsreferendum sui generis unterstellt. Im Unterschied zur seinerzeitigen Kenntnislage vor dem geplanten Beitritt zum EWR besteht heute ein vertieftes Wissen über die Funktionsweise der bilateralen Verträge, sodass die supranationale Qualität des InstA als deren institutionelle Fortentwicklung zuverlässiger eingeordnet werden kann.

I. Rechtliche Ausgangslage

II. Obligatorisches Staatsvertragsreferendum (Art. 140 Abs. 1 Bst. b BV).

III. Obligatorisches Staatsvertragsreferendum sui generis

IV. Obligatorisches Staatsvertragsreferendum gemäss

Art. 140 Abs. 1 Bst. b bis BV (neu)

V. Zusammenschau zur Unterstellung unter das obligatorische Referendum. 225

Zitiervorschlag: Andreas Glaser, Das Institutionelle Abkommen Schweiz-EU ein Fall für das obligatorische Staatsvertragsreferendum?, in: sui-generis 2020, S. 210

URL: sui-generis.ch/131

DOI: https://doi.org/10.21257/sg.131

* Andreas Glaser (andreas.glaser@rwi.uzh.ch), Prof. Dr. iur., Lehrstuhlinhaber für Staats-, Verwaltungs- und Europarecht unter besonderer Berücksichtigung von Demokratiefragen an der Universität Zürich/Vorsitzender der Direktion des Zentrums für Demokratie Aarau (ZDA). Der Beitrag basiert auf einem im Auftrag von Dr. Andreas Honegger, Prof. Dr. Martin Janssen und Gregor Rutz verfassten Gutachten. 


\section{Rechtliche Ausgangslage}

1 Das «Abkommen zur Erleichterung der bilateralen Beziehungen zwischen der Europäischen Union und der Schweizerischen Eidgenossenschaft in den Bereichen des Binnenmarkts, an denen die Schweiz teilnimmt» - kurz Institutionelles Abkommen (InstA) - bedürfte zu seinem Inkrafttreten zunächst der Ratifikation durch den Bundesrat (Art. 184 Abs. 2 Satz 1 BV)1. Daraufhin müsste der Bundesrat das InstA dem Parlament zur Genehmigung unterbreiten (Art. 184 Abs. 2 Satz 2 BV). In einem nächsten Schritt befände demnach die Bundesversammlung über die Genehmigung des InstA (Art. 166 Abs. 2 BV, Art. 24 Abs. 2

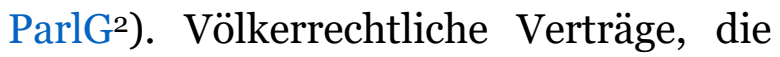
dem Referendum unterliegen, genehmigt sie in der Form eines Bundesbeschlusses (Art. 24 Abs. 3 Satz 1 ParlG). Das Parlament hätte dabei also zu entscheiden, ob es den Bundesbeschluss dem obligatorischen oder dem fakultativen Referendum unterstellt.

2 Im Folgenden wird anhand der massgeblichen verfassungsrechtlichen Bestimmung in Art. 140 Abs. 1 Bst.b BV (dazu II.), der bisherigen Staatspraxis (dazu III.) und möglicher künftiger verfassungsrechtlicher Entwicklungen untersucht (dazu IV.), ob der Genehmigungsbeschluss zum InstA dem obligatorischen Referendum zu unterstellen ist. Die Untersuchung wird durch eine $\mathrm{Zu}-$ sammenschau der Überlegungen abgerundet (dazu V.).

\footnotetext{
Bundesverfassung der Schweizerischen Eidgenossenschaft vom 18. April 1999 (BV; SR 101).

2 Bundesgesetz über die Bundesversammlung vom 13. Dezember 2002 (ParlG; SR 171.10).
}

\section{Obligatorisches Staatsvertragsrefe- rendum (Art. 140 Abs. 1 Bst. b BV)}

3 Im Wege des obligatorischen Referendums wird Volk und Ständen gemäss Art. 140 Abs. 1 Bst. b BV der Beitritt zu supranationalen Gemeinschaften unterbreitet.

\section{Beitritt zu supranationalen Gemeinschaften}

4 Der Begriff des «Beitritts» ist nicht eng als Eintritt in eine bestehende Gemeinschaft zu verstehen, sondern erfasst mit Blick auf den Zweck der Vorschrift jede hinreichend intensive Form der Beteiligung. 3 Daniel Thürer und Oliver Diggelmann halten in diesem Sinne dafür: «Denkbar wäre etwa, im Fall einer weiteren Integration der Schweiz in den EUBinnenmarkt eine Unterstellung unter den EuGH als Beitritt zu einer supranationalen Gemeinschaft zu betrachten. Zwar würde es sich formal nicht um einen Beitritt handeln. Die Auswirkungen wären angesichts der umfassenden $\mathrm{Zu}$ ständigkeiten des EuGH, seiner Unabhängigkeit und seiner allgemeinen Bedeutung für die EU jedoch beitrittsähnlich.»4

5 Im Hinblick auf die Neuordnung des Staatsvertragsreferendums im Jahr 1977 unternahm der Bundesrat den Versuch einer Definition der supranationalen Gemeinschaft: 5 «Supranationale Organi-

3 Etienne Grisel, Initiative et référendum populaires, 3. Aufl., 2004, Rz. 791.

4 Daniel Thürer/Oliver Diggelmann, in: St. Galler Kommentar, Band I und Band II, 3. Aufl. 2014, Rz. 23 zu Art. 140 BV.

5 Botschaft vom 23. Oktober 1974 über die Neuordnung des Staatsvertragsreferendums, BBl 1974 II 1133, 1156. Siehe auch Botschaft vom 18. Mai 1992 zur Genehmigung des Abkommens über den Europäischen Wirtschaftsraum, 
sationen» im Sinne unseres Vorschlages sind Organisationen

- mit Organen aus unabhängigen Personen, die nicht an Instruktionen der Regierung ihres Heimatstaates gebunden sind;

- mit Organen, die ihre Befugnisse durch Mehrheitsbeschluss und nicht nach dem Einstimmigkeitsprinzip ausüben;

- deren Entscheide direkt in Kraft treten und für Einzelpersonen unmittelbar verbindlich sind;

- deren materielle Befugnisse relativ umfassend sind.

Nach allgemeiner Auffassung erfüllt die Europäische Union als einzige Organisation die Merkmale einer supranationalen Gemeinschaft. ${ }^{6}$ Kennzeichnend für die supranationale Gemeinschaft EU ist heute, dass sich ihr Aufgabenbereich auf ein breites Spektrum von Tätigkeiten erstreckt, ihre Organe teilweise ohne Bindung an Instruktionen der Mitgliedstaaten entscheiden, instruierte Organe teilweise mit Mehrheit entscheiden, Beschlüsse der Organe nicht nur Mitgliedstaaten verpflichten, sondern unmittelbare Rechtswirkungen für Bürgerinnen und Bürger und Vorrang vor dem nationalen Recht haben können, sowie eine eigene Gerichtsbarkeit.7

BBl 1992 IV 1, 539; Thürer/Diggelmann (Fn. 4), Rz. 21; siehe auch Yvo Hangartner/Andreas Kley, Die demokratischen Rechte in Bund und Kantonen der Schweizerischen Eidgenossenschaft, 2000, Rz. 1141.

6 Siehe nur Giovanni Biaggini, in: BV Kommentar, 2. Aufl. 2017, Rz. 7 zu Art. 140; Matthias Oesch, Europarecht, 2. Aufl. 2019, Rz. 96.

7 Tobias Jaag/Julia Hänni, Europarecht, 4. Aufl. 2015, Rz. 107.
7 Die Supranationalität des Unionsrechts zeichnet sich durch dessen unmittelbare Geltung in den Mitgliedstaaten, dessen Vorrang vor dem nationalen Recht, die Eignung zur unmittelbaren Berechtigung oder Verpflichtung von Einzelpersonen und Behörden, den Erlass im Wege unabhängiger Beschlussfassung und bei Abhängigkeit von den Mitgliedstaaten nach dem Mehrheitsprinzip sowie die obligatorische Kontrolle durch den Europäischen Gerichtshof (EuGH) aus. ${ }^{8}$

\section{Europäischer Wirtschaftsraum (EWR) als Präzedenzfall}

8 Als erstmaliger Anwendungsfall für das obligatorische Staatsvertragsreferendum unter dem Titel des Beitritts zu einer supranationalen Gemeinschaft wurde der Europäische Wirtschaftsraum (EWR) diskutiert. Zur Begründung des supranationalen Charakters angeführt wurden die automatische Übernahme weiterentwickelten Unionsrechts, die unmittelbare Anwendbarkeit des EWR-Rechts, der Vorrang des EWR-Rechts, der weitreichende materielle Inhalt des Abkommens sowie die Schaffung unabhängiger Organe (EFTA-Gerichtshof und EFTAÜberwachungsbehörde). 9 Nach anderer Auffassung sah das EWR-Abkommen weder die Übertragung von Rechtsetzungsbefugnissen und Hoheitsrechten vor, noch hätte es eine eigene, autonome Rechtsordnung geschaffen, wobei der EFTA-Überwachungsbehörde und dem EFTA-Gerichtshof immerhin supranationale Elemente zugeschrieben wurden. ${ }^{10}$

8 Werner Schroeder, Grundkurs Europarecht, 6. Aufl. 2019, § 5 Rz. 3.

9 Siehe dazu Grisel (Fn. 3), Rz. 789 f.; Hangartner/ Kley (Fn. 5), Rz. 1143.

10 Babette Brunner, Das Staatsvertragsreferendum: Ein Volksrecht im Wandel, 2014, S. 59 f. 
9 Der Bundesrat schlug einen argumentativen Mittelweg ein. Einerseits wies er auf die im EWR-Abkommen enthaltenen supranationalen Elemente wie die Einrichtung der EFTA-Überwachungsbehörde und den EFTA-Gerichtshof hin. ${ }^{11}$ Die Supranationalität des EFTAGerichtshofs machte der Bundesrat an dessen Kompetenzen zur Streitschlichtung und zur nicht verbindlichen Auslegung von Bestimmungen des EWRAbkommens auf Anfrage staatlicher Gerichte fest. Andererseits seien nicht alle Voraussetzungen für die Annahme einer supranationalen Gemeinschaft erfüllt, weshalb das ordentliche obligatorische Staatsvertragsreferendum nicht zur Anwendung gelange. ${ }^{12}$ Hieraus zog der Bundesrat den Schluss, das EWRAbkommen sei dem obligatorischen Staatsvertragsreferendum sui generis ${ }^{13}$ zu unterwerfen. ${ }^{14}$

Auch bezüglich der Assoziierung der Schweiz an Schengen und Dublin stellte der Bundesrat Überlegungen zum supranationalen Charakter des Abkommens an, lehnte dessen Supranationalität aber letztlich ab. ${ }^{15}$ Unter den gegenwärtigen Bedingungen und aus heutiger Sicht wird die Supranationalität von Schengen/ Dublin in der Literatur hingegen teilweise bejaht, da die Schweiz verpflichtet sei, Weiterentwicklungen des EU-Rechts in diesen Bereichen kurzfristig $\mathrm{zu}$ übernehmen und das Abkommen relativ

\footnotetext{
Botschaft (Fn. 5), BBl 1992 IV 1, 540.

Ebenda, S. 541.

Dazu unten III.

Botschaft (Fn. 5), BBl 1992 IV 1, 541.

Botschaft vom 1. Oktober 2004 zur Genehmigung der bilateralen Abkommen zwischen der Schweiz und der Europäischen Union, einschliesslich der Erlasse zur Umsetzung der Abkommen (Bilaterale II), BBl 2004 5965, 6289.
}

weitgehende Auswirkungen auf den schweizerischen Föderalismus habe. ${ }^{16}$

\section{Einordnung des InstA anhand der bundesrätlichen Kriterien}

11 Unter Heranziehung der in II.1. eingeführten Definition einer supranationalen Organisation wird im Folgenden der Charakter des InstA eingeordnet. Mangels Gründung einer gesonderten supranationalen Gemeinschaft geht es darum, ob das InstA beitrittsähnliche Wirkungen zur Folge hat.

\section{a) Unabhängige Organe}

Mit dem Schiedsgericht und dem EuGH sind im InstA zwei unabhängige Organe sowie zwei unabhängige Vertreter der Vertragsparteien (die Europäische Kommission und der schweizerische Bundesrat) vorgesehen, deren Mitglieder nicht an Instruktionen von Seiten der Vertragsstaaten gebunden sind. Im Ausgangspunkt überwacht die Europäische Kommission die Anwendung der Verträge durch europäische und mitgliedstaatliche Behörden, während Schweizer Behörden die Anwendung in der Schweiz überwachen. ${ }^{17}$ Somit ist keine gemeinsame supranationale Überwachungsbehörde vorgesehen. Im Interesse einer gemeinsamen Überwachung bestehen sektorielle Gemischte Ausschüsse, die in Fällen angeblich mangelhafter Anwendung der Abkommen von beiden Vertragsparteien in klassisch diplomatischer Weise befasst werden können. ${ }^{18}$

13 Aufgrund der verfahrensrechtlichen Struktur könnte die Kommission jedoch

16 Thürer/Diggelmann (Fn. 4), Rz. 26.

17 Art. 6 Abs. 1, Art. 7 Abs. 1 InstA.

18 Art. 7 Abs. 2 InstA. 
auch im Rahmen des InstA im Verhältnis zur Schweiz faktisch die Rolle einer Überwachungsbehörde übernehmen. Die Kommission ist das supranationale Organ der EU schlechthin, ${ }^{19}$ der unter anderem die Überwachung der Anwendung der Verträge sowie des sekundären Rechts im Wege des Vertragsverletzungsverfahrens (Art. 258 ff. AEUV2o) obliegt.

14 Kommt es im sektoriellen Gemischten Ausschuss zu keiner einvernehmlichen Lösung, kann jede Vertragspartei verlangen, dass ein Schiedsgericht den Streitfall entscheidet. ${ }^{21}$ Die Kommission als Vertreterin der Vertragspartei EU kann demnach einseitig und ohne Zustimmung der Schweiz an das Schiedsgericht gelangen. Wirft der Streitfall eine Frage betreffend die Auslegung oder Anwendung einer Bestimmung auf, die im Unionsrecht vorkommt, und ist deren Auslegung für die Streitbeilegung relevant und für seine Entscheidung notwendig, ${ }^{22}$ so ruft das Schiedsgericht den EuGH an. ${ }^{23}$ Das Urteil des EuGH ist für das Schiedsgericht verbindlich. ${ }^{24}$

Abgesehen von der Thematisierung im Gemischten Ausschuss und der insoweit rein formalen Zwischenschaltung des Schiedsgerichts ähnelt die Ausgestaltung dem Vertragsverletzungsverfahren, das die Kommission im Rahmen der EU zu-

19 Jaag/Hänni (Fn. 7), Rz. 1766, 1776.

20 Vertrag über die Arbeitsweise der Europäischen Union (AEUV).

21 Art. 10 Abs. 2 InstA.

22 Siehe zum Vorbild des Schiedsgerichts im Assoziierungsabkommen EU - Ukraine: Glaser/Dörig, Zur Streitbeilegung in den Bilateralen Abkommen Schweiz - EU, in: Schweizerisches Jahrbuch für Europarecht 2017/2018, 2018, S. 462 f.

23 Art. 10 Abs. 3 Satz 1 InstA; Art. III.9 Abs. 1 Protokoll über das Schiedsgericht.

24 Art. 10 Abs. 3 Satz 2 InstA. lasten von Mitgliedstaaten anstrengen kann. 25 Sowohl nach dem Vertragsverletzungsverfahren als auch nach dem InstA kann die Kommission eine ihrer Ansicht nach unionsrechtswidrige Verhaltensweise eines EU-Mitgliedstaats beziehungsweise der Schweiz eigenständig einer Entscheidung durch den EuGH zuführen. Somit ist die Kommission auch Überwachungsbehörde für die Schweiz («Hüterin der Verträge»). ${ }^{26}$

16 Aufgrund der Konstruktion des Streitbeilegungsverfahrens mit einseitiger Anrufungsmöglichkeit durch die Kommission sowie obligatorischer und verbindlicher Entscheidung durch den EuGH wird ein funktionales Äquivalent zum Vertragsverletzungsverfahren geschaffen. Soweit das Schiedsgericht ohne Einbezug des EuGH entscheidet, fungiert das Gremium selbst als von den Instruktionen der Vertragsparteien unabhängiges Organ. Die Schiedssprüche sind endgültig und bindend für alle Parteien. ${ }^{27}$ Die Parteien müssen die Entscheidungen unverzüglich umsetzen. ${ }^{28}$

17 Soweit der EuGH in Fällen mit Bezug zum Unionsrecht das für das Schiedsgericht verbindliche Urteil fällt, ${ }^{29}$ ist auch der EuGH selbst als unabhängiges Organ anzusehen. Im Rahmen der EU (vgl.

$25 \overline{\text { Anders Astrid Epiney, Der Entwurf des Instituti- }}$ onellen Abkommens Schweiz - EU, Jusletter 17. Dezember 2018, Rz. 45, wonach eine Ähnlichkeit zum Vorabentscheidungsverfahren bestehen soll.

26 Carl Baudenbacher, Rechtsgutachten zur Streitentscheidungsregelung des InstA zu Handen der Kommission des Nationalrates für Wirtschaft und Abgaben WAK, 2019, S. 41.

27 Art. IV.2 Abs. 2 Satz 2 Protokoll über das Schiedsgericht.

28 Art. IV.2 Abs. 5 Protokoll über das Schiedsgericht.

29 Art. 10 Abs. 3 Satz 2 InstA. 
Art. 19 EUV) ist die Unabhängigkeit des EuGH als oberstes Rechtsprechungsorgan anerkannt. 30

\section{b) Nach Mehrheitsregel entscheidende Organe}

Soweit es sich um nicht unabhängige, sondern von Instruktionen der Vertragsstaaten abhängige Organe handelt, müssen diese mit Mehrheit entscheiden, damit Supranationalität angenommen werden kann. Typisch hierfür ist das ordentliche Gesetzgebungsverfahren in der EU gemäss Art. 294 AEUV. ${ }^{11}$ Dabei entscheidet das Europäische Parlament mit einfacher Mehrheit der abgegebenen Stimmen oder der Abgeordneten. Der Rat der Europäischen Union entscheidet mit qualifizierter Mehrheit. Rechtsakte der EU in Form der Verordnung und der Richtlinie können demnach gegen den Willen eines oder mehrerer Mitgliedstaaten verabschiedet werden.

Es ist fraglich, ob das Verfahren der Rechtsübernahme im InstA supranationalen Charakter wie im Rahmen einer EU-Mitgliedschaft annimmt oder weiterhin das statische Konzept der bisherigen bilateralen Verträge teilt. Weder findet derzeit eine automatische Rechtsübernahme von neuem Unionsrecht durch die Schweiz statt, noch besteht eine Verpflichtung der Schweiz zur dynamischen Übernahme neu erlassener Rechtsakte der EU. 32

30 Peter Michael Huber, in: Streinz (Hrsg.), EUV/AEUV, 3. Aufl. 2018, Rz. 28 zu Art. 19 EUV (Gerichtshof der Europäischen Union).

${ }^{31}$ Siehe dazu Oesch, Europarecht (Fn. 6), Rz. 488.

32 Matthias Oesch, Die bilateralen Abkommen Schweiz - EU und die Übernahme von EU-Recht, AJP 2017, S. 639 ff.
20 Das InstA sieht hingegen ein Verfahren zur Übernahme von Rechtsakten der EU durch die Schweiz auf dem Gebiet der betroffenen Abkommen vor.33 Im Unterschied zur geltenden Rechtslage im Rahmen der bilateralen Verträge wäre die Schweiz neu verpflichtet, der Anpassung der Abkommen zuzustimmen.34 Die Schweiz hat die Erfüllung der Pflicht zur Rechtsübernahme der EU über den sektoriellen Gemischten Ausschuss unverzüglich zu notifizieren.35 Verweigert die Schweiz die Anpassung eines Abkommens, entsteht dementsprechend ein Streitfall, auf den das Streitbeilegungsverfahren anwendbar ist. 36

21 Nachdem die EU einen einschlägigen Rechtsakt verabschiedet hat, ist die Schweiz verpflichtet, im zuständigen sektoriellen Gemischten Ausschuss gemeinsam mit der EU einen Beschluss zu fassen beziehungsweise die Anpassung des Abkommens vorzuschlagen, um den EURechtsakt darin zu integrieren. 37 Beschlüsse des sektoriellen Gemischten Ausschusses zur Integration von EURechtsakten in den bilateralen Rechtsbestand treten sofort, Revisionen am Tag ihrer Unterzeichnung durch die Vertragsparteien in Kraft, aber nicht vor dem Zeitpunkt des Inkrafttretens in der EU. 38

22 Bei diesem Mechanismus handelt es sich nicht um eine automatische Rechtsübernahme, da die Schweiz über jede Anpas-

Art. 1 Abs. 3 Spstr. 1, Art. 5 InstA.

4 Epiney (Fn. 25), Rz. 21.

35 Art. 14 Abs. 2 Satz 1 InstA.

36 Eidgenössisches Departement für auswärtige Angelegenheiten (EDA), Erläuterungen zum Institutionellen Abkommen Schweiz - EU, 2019, S. 7 .

37 Art. 13 Abs. 1 und 2 Satz 1 InstA.

38 Art. 13 Abs. 2 Satz 2 InstA. 
sung einzeln in dem verfassungsrechtlich vorgegebenen Verfahren einschliesslich der Wahrung des Referendumsrechts befinden kann. 39 Es stellt sich die Frage, ob die dynamische Rechtsübernahme noch dem klassischen völkerrechtlichen Ansatz verhaftet ist oder schon die supranationalen Züge des EU-Gesetzgebungsverfahrens annimmt. Für letztere Sichtweise sprechen zwei Argumente.

Die dynamische Rechtsübernahme nähme keinen supranationalen Charakter an, wenn eine Pflichtverletzung nicht systematisch und ernsthaft sanktioniert würde. Genau dies ist jedoch im InstA vorgesehen. Eine Ablehnung der Übernahme des Rechtsakts durch das Parlament oder in der Volksabstimmung führt nicht zu einer Entbindung von der Übernahmepflicht, sondern stellt vielmehr deren Verletzung dar. Diese kann im Wege eines Streitbeilegungsverfahrens thematisiert werden 40 oder als Anlass für die Ergreifung von Ausgleichsmassnahmen durch die EU zulasten der Schweiz dienen.

Zum anderen spricht der starke Einbezug der Schweiz bei der Ausarbeitung der Rechtsakte für den supranationalen Charakter der Rechtsübernahme. Die EU informiert die Schweiz, sobald mit der Ausarbeitung eines relevanten Rechtsakts begonnen wird und zieht Sachverständige der Schweiz informell gleichermassen zu Rate wie Sachverständige der Mitgliedstaaten. ${ }^{41}$ Handelt es sich um delegierte Rechtsakte gemäss Art. 290 AEUV, mit denen die Kommission

39 EDA, Institutionelles Abkommen Schweiz - EU: Das Wichtigste in Kürze, S. 2.

40 Epiney (Fn. 25), Rz. 26.

41 Art. 12 Abs. 1 InstA.
Rechtsakte ohne Gesetzescharakter mit allgemeiner Geltung zur Ergänzung oder Änderung bestimmter nicht wesentlicher Vorschriften eines Gesetzgebungsakts erlassen darf, gewährt die Kommission der Schweiz die grösstmögliche Teilnahme an der Ausarbeitung ihrer Vorschläge. 42 Vergleichbares gilt bei Durchführungsrechtsakten gemäss Art. 291 AEUV, mit denen die Kommission den einheitlichen Vollzug von Unionsrechtsakten regelt.43 Die Schweizer Sachverständigen wirken wie diejenigen der EU-Mitgliedstaaten im Rahmen der Komitologieausschüsse an der Verabschiedung von Durchführungsrechtsakten mit. 44

\section{c) Direkte Anwendbarkeit und Verbindlichkeit für Individuen und Behörden}

\section{aa) Geltung und Anwendungsvor- rang des Sekundärrechts}

25 Mit der Zustimmung zum InstA fände keine Ausdehnung des EU-Rechtsbestandes, wie beispielsweise der Grundfreiheiten, statt. 45 Auch ist nicht vorgesehen, dass das EU-Recht in der schweizerischen Rechtsordnung unmittelbar gilt, jede Übernahme von Unionsrecht durch die Schweiz erfordert vielmehr einen selbstständigen Entscheid der Schweiz. ${ }^{4}$ Als Inbegriff einer supranationalen Erlassform wird demgegenüber die EUVerordnung (Art. 288 Abs. 2 AEUV) angesehen, da sie mit Inkrafttreten Vorrang vor dem mitgliedstaatlichen Recht besitzt

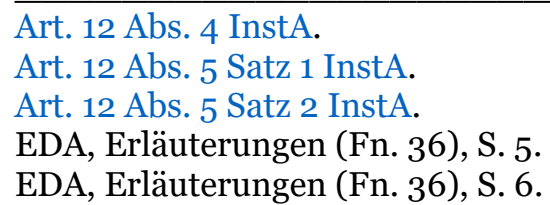


und sich Individuen wie Organe auf eine Verordnung berufen können. 47

Soweit der Anwendungsbereich des InstA reicht, entfalten allerdings jedenfalls einzelne Bestimmungen der in Bezug genommenen bilateralen Verträge direkte Anwendbarkeit und sind für Behörden wie Individuen verbindlich. Aufgrund des in der Schweiz herrschenden monistischen Systems bei der Integration völkerrechtlicher Bestimmungen in die nationale Rechtsordnung können Rechtsakte der EU, die im Wege der bilateralen Verträge übernommen wurden, direkt anwendbar sein sowie unmittelbar Rechte und Pflichten begründen. ${ }^{8}$ Dies ist bei zahlreichen Bestimmungen der in Bezug genommenen bilateralen Verträge der Fall. Praktisch am bedeutsamsten dürften die Aufenthaltsansprüche zugunsten von Einzelpersonen nach dem FZA sein. Das Bundesgericht nimmt bereits heute an, dass die bilateralen Abkommen und die darin als verbindlich erklärten Anhänge aufgrund des monistischen Systems mit ihrer Ratifizierung automatisch Teil der schweizerischen Rechtsordnung werden und keiner besonderen Umsetzung mehr im nationalen Recht bedürfen, falls sie hinreichend konkretisiert sind, um in einem Einzelfall direkt angewendet werden $\mathrm{zu}$ können («selfexecuting»). 49

47 Jaag/Hänni (Fn. 7), Rz. 2109; Oesch, Europarecht (Fn. 6), Rz. 424.

48 Siehe dazu bereits Andreas Glaser, Umsetzung und Durchführung des Rechts der Bilateralen Verträge in der Schweiz, in: Glaser/Langer (Hrsg.), Die Verfassungsdynamik der europäischen Integration und demokratische Partizipation, 2015, S. 136.

49 BGE 138 II 42 E. 3.2 S. 46 f.
27 Flankiert wird die für das Unionsrecht typische unmittelbare Anwendbarkeit durch den potenziellen Vorrang des InstA und der in Bezug genommenen bilateralen Verträge vor dem gesamten nationalen Recht, einschliesslich der Bundesverfassung. Zwar fehlt es an einer Bestimmung der Rangfolge im InstA und auch im nationalen Recht. Die Politik überlässt die Handhabung der Rangfrage somit der Rechtspraxis. Das Bundesgericht vertritt aber immerhin bereits heute die Auffassung, das FZA gehe aufgrund seines menschenrechtlichen Charakters analog zur EMRK dem nationalen Recht vor.50 Die Bezugnahme des Bundesgerichts auf die für die Charakterisierung des Unionsrechts als supranationales Recht konstitutiven EuGH-Urteile und deren Übertragung auf das bilaterale Verhältnis Schweiz-EU legt zudem nahe, dass es den bilateralen Verträgen bereits heute supranationalen Charakter zuspricht. Dies muss erst recht gelten, wenn die bilateralen Verträge mit einem wirksameren Überwachungs- und Durchsetzungsmechanismus ausgestattet würden, als dies bislang der Fall ist. ${ }^{11}$ Aus der Kombination von direkter Anwendbarkeit und Anwendungsvorrang der vom InstA erfassten Bestimmungen ergibt sich eine mit Blick auf den Beitritt zur EU ähnliche Wirkung.

\section{bb)Direkt anwendbare Bestimmungen im InstA}

28 Überdies enthält das InstA selbst direkt anwendbare Bestimmungen. Zwar wird auf der einen Seite argumentiert, bei den beihilfenrechtlichen Bestimmungen hand-

50 BGE 142 II 35 E. 3.2 S. 39 f. Siehe auch Glaser (Fn. 48), S. 146.

51 Vgl. zu diesem Gedanken BGE 139 I 16 E. 5.2.3 S. $30 \mathrm{f}$. 
le es sich um nicht direkt anwendbare Grundsätze, welche folglich nicht justiziabel seien. ${ }^{2}$ Zugleich wird aber auf der anderen Seite eingeräumt, dass die materiellen Bestimmungen betreffend die Staatsbeihilfen im Bereich des Luftverkehrsabkommens, wo bereits heute eine Beihilfenüberwachung stattfindet, direkt anwendbar sind.53

Von besonderer Relevanz ist das Verbot staatlicher Beihilfen.54 Dieses entspricht dem Regelungsvorbild des Art. 107 AEUV.55 Insgesamt lehnt sich das Beihilfenregime eng an dasjenige innerhalb der EU an. ${ }^{6}$ So dürfen geplante Beihilfenregelungen oder einzelne Beihilfenvorhaben erst umgesetzt werden, wenn die Überwachungsbehörde im Rahmen des einzurichtenden Notifizierungsverfahrens abschliessend entschieden hat. 57 Das in der ganz ähnlich formulierten unionsrechtlichen Bestimmung (Art. 108 Abs. 3 Satz 3 AEUV) verankerte, mit unmittelbarer Anwendbarkeit und Vorrang vor dem nationalen Recht ausgestattete Durchführungsverbot wird als Ausprägung maximaler Supranationalität bezeichnet. 58

EDA, Erläuterungen (Fn. 36), S. 4, 10. Siehe ausserdem Epiney (Fn. 25), Rz. 43.

54 Art. 8A Abs. 2 Bst. a InstA.

55 Siehe dazu Philipp Zurkinden, Institutionelles Abkommen - Rechtsgutachten zuhanden der WAK-N; Fragen zu Staatsbeihilfen und zum Freihandelsabkommen $\mathrm{CH}$ - EU sowie $\mathrm{zu}$ weiteren Fragen, 2019, Rz. 33; Ylber Hasani, Die Beihilfenkontrolle in der Europäischen Union, Jusletter 29. April 2019, Rz. 37.

56 EDA, Das Wichtigste in Kürze (Fn. 39), S. 11.

57 Art. 8B Abs. 3 InstA.

58 Volkmar Götz, Staatliche Beihilfen, in: Dauses (Hrsg.), Handbuch des EU-Wirtschaftsrechts, Werkstand: 49. EL November 2019, Rz. 6 f.
30 Die Beschränkung der direkten Anwendbarkeit auf das Luftverkehrsabkommen gilt wohl nur vorläufig, da die EU den Abschluss neuer Marktzugangsabkommen, wie beispielsweise eines Strommarktabkommens, von der Anwendbarkeit einer effektiven Beihilfenüberwachung nach dem Vorbild des Unionsrechts abhängig macht.59 Zudem enthält der Anhang des InstA einen Beschlussentwurf, wonach die Beihilfenregelung im Rahmen des Streitbeilegungsverfahrens neu auch in Bezug auf das Freihandelsabkommen 1972 zur Anwendung gebracht werden soll.6o

\section{d) Relativ umfassende materielle Befugnisse}

31 Schliesslich setzt die Supranationalität relativ umfassende materielle Befugnisse der mit Kompetenzen ausgestatteten Organe voraus. Das InstA findet Anwendung auf die fünf Abkommen der Bilateralen I aus dem Jahr 1999 und künftige Marktzugangsabkommen. ${ }^{61}$ Ein Vergleich mit der EU-Mitgliedschaft ergibt daher, dass die materiellen Befugnisse eher weniger umfangreich sind. So wäre die Schweiz weiterhin weder an der gemeinsamen Währung noch an der Gemeinsamen Sicherheits- und Aussenpolitik beteiligt. Gewisse weitere Bereiche sind von vornherein ausgeklammert.

32 Nicht explizit ausgenommen ist hingegen die Unionsbürgerrichtlinie 2004/38. Diese könnte möglicherweise einen prominenten künftigen Anwendungsfall für das Streitbeilegungsverfahren in Bezug auf die dynamische Rechtsübernahme bil-

59 EDA, Erläuterungen (Fn. 36), S. 10.

60 Vgl. Ebenda, S. 11. Zur rechtlichen Tragweite näher Hasani (Fn. 55), Rz. 39 f.

61 Art. 2 InstA. 
den. ${ }^{62}$ Hier läge wohl das grösste Risiko für materiell-rechtliche Überraschungen. ${ }^{63}$ Ähnliches könnte für die Revision der Verordnung (EG) Nr. 883/2004 zur Koordinierung der Systeme der sozialen Sicherheit gelten. In Randbereichen unklar ist auch, inwieweit die Flankierenden Massnahmen gegenüber Rechtsmitteln immunisiert wären. Eine Einordnung anhand von Sachbereichen und Kompetenzen führt in diesem Zusammenhang jedoch nicht weiter. So ist die Kompetenzverteilung in Bundesstaaten je unterschiedlich und auch die Europäische Union verfügt bei weitem nicht über umfassende Kompetenzen.

33 Es ist gerade Ausdruck von Supranationalität, dass sich - anders als dies noch bei den bilateralen Verträgen I und II der Fall war - die genauen materiellen Befugnisse von Organen der Europäischen Union im Rahmen der Vertragsbeziehungen nicht prognostizieren lassen.

\section{Zwischenergebnis}

34 Der Abschluss des InstA hätte folglich mit Blick auf die massgeblichen Gesichtspunkte ähnliche Wirkungen wie der Beitritt zu einer supranationalen Gemeinschaft. Die Mitwirkung der Schweiz in dem im InstA vorgesehenen institutionellen Rahmen kommt dem Beitritt zu einer supranationalen Organisation gemäss Art. 140 Abs. 1 Bst. b BV gleich. Der Genehmigungsbeschluss ist demnach dem obligatorischen Referendum zu unterstellen.

$6 2 \longdiv { \text { Siehe auch EDA (Fn. 39), S. 4. Ausführlich dazu } }$ Astrid Epiney/Sian Affolter, Das Institutionelle Abkommen und die Unionsbürgerrichtlinie, Jusletter 11. März 2019, Rz. 30 ff.

63 «Internes Papier des Bundes zeigt: EU-Richtlinie verschärft Konflikt mit Ausschaffungsartikel», nzz.ch vom 24. Januar 2019.

\section{Obligatorisches Staatsvertrags- referendum sui generis}

35 Würde der supranationale Charakter der Einbindung der Schweiz durch das InstA entgegen der hier vertretenen Ansicht verneint, wäre die Frage aufzuwerfen, ob der Genehmigungsbeschluss nicht unter einem anderen Titel dem obligatorischen Referendum zu unterstellen wäre. 64

\section{Ungeschriebenes Recht in der Staatspraxis}

36 Heute ist das obligatorische Referendum für völkerrechtliche Verträge mit verfassungsmässigem Charakter (Referendum sui generis) als ungeschriebenes Verfassungsrecht anerkannt. ${ }^{65}$ Der Bundesrat spricht insoweit von einem obligatorischen Staatsvertragsreferendum sui generis. ${ }^{66}$ Ein Staatsvertrag ist immer dann dem ausserordentlichen Referendum zu unterstellen, wenn er entweder tiefgreifend in die verfassungsrechtliche Ordnung eingreift, eine grundsätzliche Änderung der schweizerischen Aussenpolitik mit sich bringt oder Gründe von grundlegender sachlicher oder politischer Bedeutung dafür sprechen. ${ }^{67}$ Es muss sich um einen Vertrag von ausserordentlicher Bedeutung handeln, der den in Art. 140 Abs. 1 Bst. b BV genannten Kategorien nahe kommt.68 Der infrage stehende Staatsvertrag muss von derartiger Bedeu-

$64 \overline{\text { Vereinzelt wird in der Literatur hingegen auch }}$ ein abschliessender Charakter von Art. 140 BV angenommen, siehe dazu Epiney/Diezig, in: Basler Kommentar Bundesverfassung, 2015, Rz. 14 zu Art. 140 BV.

65 Botschaft vom 15. Januar 2020 zum obligatorischen Referendum für völkerrechtliche Verträge mit Verfassungscharakter, BBl $20201243,1247$.

66 Botschaft (Fn. 15), BBl 2004 5965, 6288.

67 Brunner (Fn. 10), S. 62.

68 Biaggini (Fn. 6), Rz. 9. 
tung sein, dass er Verfassungsrang besitzt. 69

\section{Präzedenzfälle}

37 Bei Staatsverträgen mit Verfassungsrang besteht inzwischen eine Tradition der Unterstellung unter das obligatorische Staatsvertragsreferendum sui generis. ${ }^{70}$ So wurden der Beitritt zum Völkerbund (1920), das Freihandelsabkommen Schweiz-EU (1972) und der Beitritt zum Europäischen Wirtschaftsraum (1992) dem obligatorischen Referendum unterstellt.

Zuletzt sah der Bundesrat die Anforderungen für ein obligatorisches Staatsvertragsreferendum sui generis mit Blick auf den EWR-Vertrag für gegeben an. ${ }^{71}$ So lagen mit dem EFTA-Gerichtshof und der EFTA-Überwachungsbehörde bereits supranationale Elemente vor. ${ }^{2}$ Darüber hinaus ist das EWR-Abkommen ein Staatsvertrag mit materiell umfassendem Anwendungsbereich. Zudem sind zahlreiche EWR-Bestimmungen unmittelbar anwendbar. Schliesslich bedingt der EWRVertrag notwendigerweise eine Anpassung des Verfassungsrechts.

39 Erörtert wurde die Unterstellung unter das obligatorische Staatsvertragsreferendum sui generis auch anlässlich der Assoziierung der Schweiz an Schengen und Dublin.73 Schengen/Dublin führte indes nach Auffassung des Bundesrates mit Blick auf Gegenstand und Tragweite nicht zu einer tiefgreifenden Änderung des Staatswesens und tangierte damit die

69 Botschaft (Fn. 15), BBl 2004 5965, 6289.

70 Brunner (Fn. 10), S. 61; Thürer/Diggelmann (Fn. 4), Rz. $24 \mathrm{ff}$.

71 Botschaft (Fn. 5), BBl 1992 IV 1, 541.

72 Siehe oben II. 2.

73 Botschaft (Fn. 15), BBl 2004 5965, 6290. verfassungsmässige Ordnung nicht. Insbesondere habe die Umsetzung der Abkommen im Rahmen der bestehenden Kompetenzen von Bund und Kantonen erfolgen können. Deshalb wurde in diesem Fall von der Unterstellung unter das obligatorische Staatsvertragsreferendum sui generis abgesehen.

\section{Herausragende Bedeutung des InstA}

40 Um das obligatorische Staatsvertragsreferendum sui generis auszulösen, müsste das InstA tiefgreifend in die verfassungsrechtliche Ordnung eingreifen, eine grundsätzliche Änderung der schweizerischen Aussenpolitik mit sich bringen beziehungsweise grundlegende sachliche oder politische Bedeutung aufweisen.

\section{a) Supranationale Elemente des InstA}

41 Das InstA weist, wenn man davon ausgeht, dass es keine supranationale Organisation ist, immerhin einige unverkennbare supranationale Elemente auf. Die Einrichtung eines Streitschlichtungsverfahrens, durch dessen Auslösung die Kommission einseitig eine Entscheidung durch den EuGH herbeiführen kann, ähnelt dem klassischen supranationalen Vertragsverletzungsverfahren im Rahmen der EU.74 Das von der Schweiz grundsätzlich $\mathrm{zu}$ übernehmende Recht wird im ordentlichen Gesetzgebungsverfahren der EU nach dem Mehrheitsprinzip beschlossen.75 Verschiedene Bestimmungen der unter dem Dach des InstA zusammengefassten sektoriellen Abkommen sind in der schweizerischen Rechtsordnung direkt anwendbar und

74 Siehe oben II. 3. a.

75 Siehe oben II. 3. b. 
geniessen nach bundesgerichtlicher Rechtsprechung Vorrang vor nationalem Recht. ${ }^{6}$ Vereinzelt trifft dies sogar auf Bestimmungen des InstA selbst zu, nämlich auf das für das Luftverkehrsabkommen anwendbare Beihilfenrecht. 77

Aufgrund zahlreicher supranationaler Wirkungsweisen im Rahmen des InstA und mit Blick auf den Präzedenzfall des EWR-Abkommens, das ebenfalls einzelne supranationale Komponenten aufwies, muss der Genehmigungsbeschluss jedenfalls dem obligatorischen Staatsvertragsreferendum sui generis unterstellt werden.

\section{b) Überlagerung der Bundesverfassung}

43 Für eine Unterstellung unter das Staatvertragsreferendum sui generis spricht ausserdem, dass verschiedene verfassungsrechtliche Bestimmungen berührt beziehungsweise sogar teilweise überlagert werden. Betroffen ist zunächst das parlamentarische Verfahren.

\section{aa) Verfahren zur Genehmigung völkerrechtlicher Verträge}

44 Bislang steht es dem Parlament frei, aus rein politischen Gründen eine Änderung der bilateralen Verträge zu genehmigen oder abzulehnen. Inskünftig unterläge das Parlament der grundsätzlichen Pflicht zur Genehmigung der Vertragsänderung. Dabei stünde es jeweils unter dem Eindruck möglicherweise drohender Ausgleichsmassnahmen. Am bedeutsamsten ist jedoch die Umsetzungsfrist von zwei Jahren. Die Bestimmungen der BV würden somit durch die Zweijahres-

Siehe oben II. 3. c. aa.

Siehe oben II. 3 . c. bb. frist zur Annahme des Beschlusses überlagert. Auch die vorläufige Anwendung der Vertragsänderung beeinträchtigt die freie Willensbildung im Parlament. Insgesamt erweist sich die verfassungsrechtliche Einhegung der dynamischen Rechtsübernahme nach gegenwärtiger Rechtslage als äusserst prekär. ${ }^{78}$ Die Beteiligung der Bundesversammlung an der Gestaltung der Aussenpolitik (Art. 166 Abs. 1 BV) scheint ohne verfahrensrechtliche Ergänzungen in Form von frühzeitigen Mitwirkungsrechten nicht mehr gesichert.

45 Der durch die materielle Pflicht zur Rechtsübernahme erzeugte Druck wie auch die -um ein weiteres Jahr auf drei Jahre verlängerte - Genehmigungsfrist beeinträchtigen das fakultative Staatsvertragsreferendum (Art. 141 Abs. 1 Bst. d BV) und die freie Willensbildung der Stimmberechtigten bei der Volksabstimmung (Art. 34 Abs. 2 BV). 79

\section{bb)Stellung und Unabhängigkeit des Bundesgerichts}

46 Überdies tangiert das Streitbeilegungsverfahren die Stellung des Bundesgerichts als oberste rechtsprechende Behörde des Bundes (Art. 188 Abs. 1 BV) und dessen Unabhängigkeit (Art. 191c BV). Dies ist zum einen darauf zurückzuführen, dass die Auslegung des europäischen Rechts durch den EuGH in anderen ihm durch die Gerichte der Mitgliedstaaten unterbreiteten Fällen auch für das Bundesgericht verbindlich ist (Art. 4 Abs. 1 InstA). Zum anderen und vor allem aber können die Exekutiven der Schweiz und der EU im Rahmen des Gemischten Aus-

78 Siehe dazu bereits Glaser (Fn. 48), S. 140.

79 Vgl. auch Oesch (Fn. 32), S. 638, $651 \mathrm{f}$. 
schusses (Art. 16 Abs. 1 InstA) darüber befinden, ob sie ein Urteil des Bundesgerichts allenfalls für unbeachtlich erklären oder zumindest der Überprüfung durch ein Schiedsgericht und damit mittelbar dem EuGH zuführen wollen (Art. 10 Abs. 3 und 5 InstA). ${ }^{80}$ Im Rahmen der EU-Mitgliedschaft behalten die nationalen Höchstgerichte demgegenüber - unter Vorbehalt der Vorlagepflicht im Rahmen des Vorabentscheidungsverfahrens (Art. 267 Abs. 3 AEUV) - die Letztentscheidungskompetenz.

Eine Aufhebung nationaler Gerichtsurteile durch den EuGH ist in den Klageverfahren nach dem AEUV nicht vorgesehen. Gegenstand der Nichtigkeitsklage (Art. 263 AEUV) können nur Akte der EU sein. Daher wäre es im Rahmen der EU allenfalls denkbar, wegen unterlassener Vorlage durch ein nationales Höchstgericht ein Vertragsverletzungsverfahren einzuleiten. ${ }^{81}$ Die Kommission macht hiervon mit Blick auf die Unabhängigkeit der Gerichte nur sehr zurückhaltend Gebrauch und schreitet nur ein, wenn die Nichtvorlage beispielsweise "auf offensichtlicher Unkenntnis oder einer bewussten Haltung» des nationalen Gerichts beruht. ${ }^{82}$

80 Vgl. auch Andreas Zünd, «Das Bundesgericht verliert seine Bedeutung», St. Galler Tagblatt vom 23. März 2019.

81 Martin Burgi, § 6 Vertragsverletzungsverfahren, in: Rengeling/Middeke/Gellermann (Hrsg.), Handbuch des Rechtsschutzes in der Europäischen Union, 3. Aufl. 2014, § 6 Rz. 41.

82 Hans-Joachim Cremer, in: Calliess/Ruffert (Hrsg.), EUV/AEUV, 5. Aufl. 2016, Rz. 28 zu Art. 258 AEUV; Nina Wunderlich, Vertragsverletzungsverfahren, in: von der Groeben/Schwarze/ Hatje (Hrsg.), Europäisches Unionsrecht, 7. Aufl. 2015, Rz. 8 zu Art. 258 AEUV.

\section{cc) Kompetenzen der Kantone}

48 In Anbetracht der möglichen Beeinträchtigung der kantonalen Kompetenzen, insbesondere auf dem Gebiet der Staatsbeihilfen, erweisen sich die klassischen Mitwirkungsmöglichen der Kantone auf dem Gebiet der Aussenpolitik (vgl. Art. 55 BV) als unzureichend. Die Freiräume der Kantone zum Erlass von selektiven Förderungsmassnahmen zugunsten ausgewählter Unternehmen werden erhebliche Einschränkungen erfahren. ${ }^{8}$ Über die in Art. 45 BV vorgesehenen Informations- und Konsultationsansprüche sowie die Pflichten zur Rücksichtnahme auf die Zuständigkeiten der Kantone und deren Interessenwahrung (Art. 54 Abs. 3 BV) hinaus wären zwingende Beteiligungsverfahren vorzusehen. Dies könnte beispielsweise durch die Vertretung der Schweiz in den Gemischten Ausschüssen durch Angehörige der kantonalen Verwaltung geschehen, falls substanzielle Kompetenzen der Kantone berührt wären, wie etwa im Bereich der direkten Steuern.

\section{Zusammenfassung}

49 Selbst wenn der Genehmigungsbeschluss zum InstA nicht dem ordentlichen obligatorischen Staatsvertragsreferendum zu unterstellen wäre, müsste er in Anbetracht der Präzedenzfälle aufgrund der im InstA enthaltenen supranationalen Elemente sowie der tiefgreifenden Einflüsse auf die verfassungsmässige Ordnung dem obligatorischen Staatsvertragsreferendum sui generis unterstellt werden.

83 Entsprechend mit Blick auf Beihilfenverbote in einzelnen Marktzugangsabkommen Matthias Oesch, Die (fehlende) Disziplinierung staatlicher Beihilfen durch Kantone, AJP 2013, S. 1337, 1347. 
IV. Obligatorisches Staatsvertragsreferendum gemäss Art. 140 Abs. 1 Bst. b bis BV (neu)

In Umsetzung der von beiden Räten gutgeheissenen Motion Caroni schlägt der Bundesrat vor, den Anwendungsbereichs des obligatorischen Referendums bei Staatsverträgen zu erweitern. ${ }^{84}$ Beabsichtigt ist die Übertragung des bislang ungeschriebenen Referendums sui generis in den geschriebenen Verfassungstext. ${ }^{85}$ Die neue Bestimmung dient als Konkretisierungshilfe zur näheren Eingrenzung des bereits heute massgeblichen verfassungsmässigen Charakters eines Staatsvertrages. ${ }^{86}$ Dem obligatorischen Referendum unterstünden gemäss Art. 140 Abs. 1 Bst. bbis BV (neu) auch völkerrechtliche Verträge, die Bestimmungen von Verfassungsrang enthalten oder deren Umsetzung eine Änderung der Bundesverfassung erfordert; wobei Bestimmungen von Verfassungsrang namentlich Bestimmungen über 1. den Bestand der Grundrechte, die Bürgerrechte und die politischen Rechte, 2. das Verhältnis von Bund und Kantonen und die Zuständigkeiten des Bundes, sowie 3. die Grundzüge der Organisation und des Verfahrens der Bundesbehörden beträfen.

Massgeblich ist danach, ob ein völkerrechtlicher Vertrag Bestimmungen von Verfassungsrang in einem der genannten Bereiche enthält. Die Beurteilung des Verfassungsranges erfolgt anhand eines materiellen Verfassungsverständnisses, wonach zur Verfassung alle Rechtssätze zählen, die als Grundlage der rechtsstaatlichen und demokratischen Staatsordnung in die Verfassung gehören und damit «verfassungswürdig» sind beziehungsweise "verfassungsmässigen Charakter» haben. ${ }^{87}$ Subsidiär kommt aber auch das formelle Verfassungsverständnis zum Tragen, indem auf die Änderungsbedürftigkeit der BV abgestellt wird.

\section{Bestand der Grundrechte, Bürger- rechte oder politische Rechte}

52 Der Mechanismus der dynamischen Rechtsübernahme beeinträchtigt durch die Pflicht zur Genehmigung der von der EU beschlossenen Rechtsakte innert drei Jahren die politischen Rechte. ${ }^{88}$ Die Rechtspflicht zur Übernahme stellt einen qualitativen Unterschied zu (politischen) Sachzwängen dar, wie sie mitunter im Umfeld von Volksentscheiden diskutiert werden. Das Recht zur Ergreifung des fakultativen Referendums (Art. 141 Abs. 1 Bst. d, Art. 136 Abs. 2 BV) und die Abstimmungsfreiheit der Stimmberechtigten (Art. 34 Abs. 2 BV, Art. 136 Abs. 2 BV) bei der Ausübung des Stimmrechts stehen unter dem latenten Druck der im InstA bei der Nichtübernahme eines Rechtsaktes vorgesehenen Ausgleichsmassnahmen. Referendums- und Stimmrecht können nicht mehr auf unbefangene Art und Weise ausgeübt werden. Dies ist ein Indiz dafür, dass das InstA Bestimmungen von Verfassungsrang enthält und deshalb unter Art. 140 Abs. 1 Bst. b bis BV (neu) fallen würde.

\footnotetext{
84 Bundesbeschluss über das obligatorische Refe-
rendum für völkerrechtliche Verträge mit Verfas-

Bundesbeschluss über das obligatorische Refe-
rendum für völkerrechtliche Verträge mit Verfassungscharakter (Entwurf), BBl 20201271.

85 Botschaft (Fn. 65), BBl 2020 1243, 1255. 86 Botschaft (Fn. 65), BBl 2020 1243, 1259.
}

87 Botschaft (Fn. 65), BBl 2020 1243, $1258 \mathrm{f}$.

88 Siehe dazu oben II. 3.b.aa. 


\section{Verhältnis von Bund und Kantonen}

53 Erfasst werden durch diesen Tatbestand völkerrechtliche Verträge, die dem Bund direkt Befugnisse einräumen, die von Verfassungswegen bisher den Kantonen zugestanden wurden.89 Das InstA sieht für den Bereich der Staatsbeihilfen die Einrichtung einer Überwachungsbehörde in der Schweiz vor. Hierbei müsste es sich zwingend um eine zentralisierte Bundesbehörde handeln, etwa nach dem Vorbild der WEKO.90 Diese würde indes nicht nur Staatsbeihilfen des Bundes, sondern insbesondere auch solche der Kantone beaufsichtigen..$^{11}$ Die bislang autonom von den Kantonen geregelten Bereiche der Staatsgarantie zugunsten der Kantonalbanken, der Unternehmensbesteuerung, der Beteiligung an Elektrizitätswerken, des Gebäudeversicherungsmonopols oder der Tourismusförderung, um nur einige zu nennen, 92 unterlägen neu einer systematischen Notifizierungspflicht und der Überwachung durch eine Bundesbehörde. Die Regelungen des InstA würden somit in verschiedenen Bereichen eine Kompetenzverlagerung auf den Bund bewirken. 93

\section{Organisation oder Zuständigkeiten der Bundesbehörden}

54 Schliesslich tangiert das InstA durch die dynamische Rechtsübernahme und die Einrichtung eines Streitbeilegungsverfahrens die Zuständigkeiten der Bundesversammlung und des Bundesgerichts.

$89 \overline{\text { Bundesamt für Justiz, Obligatorisches Referen- }}$ dum für völkerrechtliche Verträge mit verfassungsmässigem Charakter; Umsetzung der Motion 15.3557 Caroni, 2018, S. 11.

90 Siehe Zurkinden (Fn. 55), Rz. 73.

91 Vgl. auch Oesch (Fn. 83), S. 1347.

92 Zurkinden (Fn. 55), Rz. 37 f., 42, 48 ff.

$93 \mathrm{Vgl}$. dazu auch Hasani (Fn. 55), Rz. 45.
55 Die Bundesversammlung wäre angesichts der Pflicht zur Übernahme der einschlägigen Unionsrechtsakte nicht mehr frei, auf der Grundlage von Art. 166 Abs. 2 BV die Genehmigung eines völkerrechtlichen Vertrages zu verweigern. Die im Hintergrund drohende Ergreifung rechtmässiger Ausgleichsmassnahmen auf der Grundlage des InstA würde die Bundesversammlung vielmehr in eine faktische Ratifikationslage versetzen.

56 Die Ausgestaltung des Streitbeilegungsverfahrens kann dazu führen, dass rechtskräftige Urteile des Bundesgerichts von der Europäischen Kommission zum Anlass für ein Streitbeilegungsverfahren genommen und damit einer Beurteilung durch den Gemischten Ausschuss und das Schiedsgericht - allenfalls unter Einbezug des EuGH - unterzogen werden könnten. Diese Möglichkeit, gerichtliche Urteile infrage zu stellen, geht über die Praxis im Vertragsverletzungsverfahren im Rahmen einer EU-Mitgliedschaft hinaus, erlegt sich doch die Kommission mit Blick auf die richterliche Unabhängigkeit besondere Zurückhaltung auf.94

\section{Zusammenfassung}

57 Das InstA enthält Bestimmungen mit verfassungsmässigem Charakter.95 So betrifft es die politischen Rechte, die $\mathrm{Zu}$ ständigkeitsverteilung zwischen Bund und Kantonen sowie die Zuständigkeiten von Bundesbehörden. Es ist somit gemäss Art. 140 Abs. 1 Bst. bis BV (neu) dem obligatorischen Staatsvertragsreferendum zu unterstellen. Da es sich hierbei um dessen Kodifikation handelt, bestätigt dies die Unterstellung unter das

94 Siehe dazu oben II. 3. a.

95 Explizit offen gelassen in der Botschaft (Fn. 65), BBl 2020 1243, 1265. 
obligatorische Staatsvertragsreferendum sui generis, falls die Neuregelung noch nicht in Kraft getreten sein sollte.

\section{Zusammenschau zur Unterstellung unter das obligatorische Referendum}

58 Nach dem geplanten EWR-Beitritt im Jahr 1992 und dem Beitritt zu Schengen/ Dublin im Jahr 2007 stellt sich mit Blick auf das InstA erneut die Frage, ob der betreffende Genehmigungsbeschluss den Beitritt zu einer supranationalen Gemeinschaft gemäss Art. 140 Abs. 1 Bst.b BV bewirkt und dieser somit dem obligatorischen Referendum zu unterstellen ist. Im Unterschied zur seinerzeitigen Kenntnislage vor dem geplanten Beitritt zum EWR besteht heute ein vertieftes Wissen über die Funktionsweise der bestehenden bilateralen Verträge, sodass die supranationale Qualität des InstA als deren institutionelle Fortentwicklung zuverlässiger eingeordnet werden kann.

59 Eine umfassende Analyse des InstA ergibt vor diesem Hintergrund, dass immer mit Blick auf den Anwendungsbereich von fünf bestimmten bilateralen Verträgen und allfälligen weiteren Marktzugangsabkommen - sämtliche anerkannten Elemente einer supranationalen Gemeinschaft gemäss Art. 141 Abs. 1 Bst. b BV erfüllt sind und somit eine beitrittsähnliche Situation gegeben ist.

Der Beitritt zum EWR war damals von den Bundesbehörden in Bezug auf die Supranationalität im Rückblick zu zurückhaltend beurteilt worden. Immerhin wurde der Beitrittsbeschluss seinerzeit aufgrund des Verfassungsrangs verschiedener Abkommensbestimmungen dem obligatorischen Staatsvertragsreferendum sui generis unterstellt. Angesichts dieses Präzedenzfalles und der im InstA angelegten Parallelen mit mehreren supranationalen Elementen müsste die Bundesversammlung das InstA jedenfalls auf der Grundlage des obligatorischen Staatsvertragsreferendums sui generis, wenn nicht sogar unter Art. 140 Abs. 1 Bst. b BV, Volk und Ständen zwingend zur Abstimmung zu unterbreiten.

61 Möglicherweise wird im Zeitpunkt der Genehmigung durch das Parlament ein weiterer Tatbestand gelten, wonach völkerrechtliche Verträge, die Bestimmungen von Verfassungsrang enthalten, dem obligatorischen Referendum zu unterstellen sind. Verschiedene Bestimmungen des InstA im Zusammenhang mit der dynamischen Rechtsübernahme, dem Streitbeilegungsverfahren und den Staatsbeihilfen weisen Verfassungsrang auf. Mithilfe von Art. 140 Abs. 1 Bst. b bis BV (neu) liesse sich das InstA demnach ebenfalls unter die Voraussetzungen des obligatorischen Staatsvertragsreferendums subsumieren. 